\title{
CIRURGIA BARIÁTRICA: PROBLEMAS ADVINDOS DO USO ABUSIVO DO ÁLCOOL E O PAPEL DO PSICÓLOGO NO TRATAMENTO
}

DOI: $10.22289 / 2446-922 X . V 4 N 3 A 8$

\author{
Gabriela Gonçalves Silva ${ }^{1}$ \\ Juliana Amorim Pacheco de Oliveira
}

\section{RESUMO}

A maioria dos pacientes submetidos à $C B$ não voltam aos níveis iniciais de morbidez. Porém, para atingir bons resultados é importante que o paciente siga as orientações médicas no pré e pós cirúrgico no que se refere ao consumo de alimentos bem como a ingestão de bebida alcóolica. Este estudo se justifica diante do elevado número de cirurgias bariátricas que vem sendo realizadas e da importância do controle pós-cirúrgico, tanto alimentar quanto relacionado ao uso de bebidas alcoólicas, para que se atinja bons resultados. O trabalho é de caráter qualitativo, partindo de uma revisão conceitual de literatura. O objetivo é alertar a respeito do uso do álcool após a realização da CB e discorrer sobre a importância do apoio do profissional psicólogo no tratamento pós-cirúrgico. Os profissionais psicólogos são instrumentos fundamentais para auxiliar os pacientes a estabelecerem metas razoáveis e realizar escolhas saudáveis de estilo de vida, com melhoras significativas tanto físicas quanto psicológicas. $O$ acompanhamento psicológico do paciente aumenta as chances de sucesso da CB, considerando que este profissional pode ajudar a reconhecer e auxiliar as psicopatologias que geralmente contribuem para o ganho de peso, tais como os transtornos fóbico-ansiosos, depressão atípica, síndrome do comer noturno e/ou transtorno da compulsão alimentar periódica.

Palavras-chave: Obesidade; Cirurgia Bariátrica; Consumo de álcool; Psicólogos.

\section{ABSTRACT}

The majority of patients submitted to BS do not return to initial levels of morbidity. However, in order to achieve good results it is important that the patient follow the medical guidelines in the pre and post surgical aspects regarding the consumption of food as well as the consumption of alcoholic beverage. This study is justified by the high number of bariatric surgeries that have been performed and the importance of postoperative control, both food and related to the use of alcoholic beverages, in order to achieve good results. The work is qualitative, starting from a conceptual review of literature. The objective is to alert about the use of alcohol after the CB and

\footnotetext{
1 Endereço eletrônico de contato: gabriela.gaby23@hotmail.com

Recebido em 20/09/2018. Aprovado pelo conselho editorial para publicação em 16/11/2018.
}

Rev. Psicol Saúde e Debate. Dez., 2018:4(3):84-95. 
to discuss the importance of the support of the professional psychologist in the post-surgical treatment. Psychologists are instrumental in helping patients to set reasonable goals and make healthy lifestyle choices with significant physical and psychological improvements. The psychological follow-up of the patient increases the chances of CB success, considering that this professional can help to recognize and help the psychopathologies that generally contribute to the weight gain, such as phobic-anxious disorders, atypical depression, nocturnal eating syndrome and / or periodic eating disorder.

Keywords: Obesity; Bariatric surgery; Alcohol consumption; Psychologists.

\section{INTRODUÇÃO}

O problema da obesidade começou a ser observado no início da década de 1990, momento em que se tornou um problema de saúde pública que necessitava de estratégias para prevenção e controle (Camargo, Barros, Antônio, \& Giglio, 2013).

Pode-se caracterizar a obesidade pelo excesso de gordura corporal resultante do desequilíbrio entre o consumo de alimentos e o gasto energético (Velásquez-Meléndez, Pimenta, \& Kac, 2004). Seus riscos se relacionam aos problemas cardiovasculares, especialmente os coronarianos, em decorrência dos altos níveis de gorduras e glicose no sangue e hipertensão arterial (Vasques, Priore, Rosado, \& Franceschini, 2010).

Diante do problema, o tratamento é recomendado para a perda e manutenção do peso. Estudos demonstram que a maioria dos pacientes submetidos ao procedimento cirúrgico não volta aos níveis iniciais de morbidez (Santo \& Cecconello, 2008). Porém, para atingir bons resultados, faz-se necessário que o paciente siga as orientações médicas no pré e pós cirúrgico (Zyger, Zanardo, \& Tomicki, 2016).

Ao falar sobre a cirurgia bariátrica (CB), Porto e Antoniassi Júnior (2017), explicam que pode ocorrer um aumento do consumo de álcool após a mesma. Há prevalência do uso de álcool em períodos pré e pós-operatórios, inferindo que a bebida alcoólica pode ter sido utilizada para suprir a falta de alimentos antes ingeridos em excesso, bem como para promover um convívio social sem constrangimentos. Em ambos os casos, pode ser a necessidade de atenção, o que origina cuidados quanto ao abuso do álcool e as suas consequências para a saúde.

Dessa forma, é importante ressaltar que sejam realizadas avaliação psicológica e entrevistas psiquiátricas para detectar psicopatologias que poderiam contribuir para o ganho de peso (transtornos fóbico-ansiosos, depressão atípica, síndrome do comer noturno e/ou transtorno da compulsão alimentar periódica). Em caso de detecção de alguma psicopatologia deve haver o acompanhamento dos profissionais especializados, integrados a uma equipe 
multidisciplinar (Fandiño, Benchimol, Coutinho, \& Apolinário, 2004). Os profissionais psicólogos são instrumentos fundamentais para auxiliar os pacientes a estabelecerem metas razoáveis e realizar escolhas saudáveis de estilo de vida, cujo o foco é conseguir melhoras físicas e psicológicas (Dobrow, Kamenetz, \& Devlin, 2002).

Dado o exposto, o presente estudo justifica-se devido ao elevado número de cirurgias bariátricas que vem sendo realizadas e também da importância do controle pós-cirúrgico, tanto alimentar quanto relacionado ao uso de bebidas alcoólicas.

Assim, o objetivo deste estudo é alertar a respeito do uso do álcool após a realização da CB e discorrer sobre a importância do apoio do profissional psicólogo no tratamento póscirúrgico. O trabalho é de caráter qualitativo, partindo de uma revisão de literatura.

\section{A OBESIDADE COMO PROBLEMA DE SAÚDE PÚBLICA}

No início da década de 1990, a obesidade começou a ser mostrar um problema que, na atualidade, se tornou uma epidemia, motivando o desenvolvimento de estudos e de critérios para definí-la, bem como estratégias para sua prevenção (Camargo et al., 2013).

As inúmeras discussões sobre a obesidade nos últimos anos resultaram em um alerta por parte da Organização das Nações Unidas (ONU), que a considerou uma doença grave, podendo produzir efeitos devastadores à saúde. A partir desta realidade, diversos estudos foram realizados no intuito de investigar os problemas relacionados ao excesso de peso (Pedroni et al., 2013).

Em 2016, a Organização das Nações Unidas para a Alimentação e a Agricultura (FAO) e a Organização Pan-Americana da Saúde (OPAS) constataram que o sobrepeso e a obesidade são particularmente preocupantes em maiores de 18 anos. Estima-se que cerca de $58 \%$ da população da América Latina e Caribe, aproximadamente 360 milhões de pessoas, estão com sobrepeso, sendo que a obesidade afeta $23 \%$ das pessoas, aproximadamente 140 milhões. Assim, o sobrepeso afeta pelo menos metade da população de todos os países da região, salvo o Haiti (38,5\%), o Paraguai $(48,5 \%)$ e a Nicarágua (49,4\%) (Panorama da Segurança Alimentar e Nutricional na América Latina e Caribe, 2016).

Ressalta-se que a obesidade é uma doença crônica que se caracteriza pelo excesso de gordura corporal, não é hereditária, e, resulta de um longo desequilíbrio entre o consumo de alimentos e o gasto energético. É um problema de saúde pública que atinge tanto os países desenvolvidos quanto os em desenvolvimento (Velásquez-Meléndez et al., 2004). 
Atualmente, observam-se inúmeras mudanças nos hábitos de vida da sociedade em geral. $\mathrm{Na}$ infância, por exemplo, ocorre o abandono do aleitamento materno e a inserção de alimentos formulados e processados. Além destes, há também a prática de jogos eletrônicos que levam as crianças a passarem muitas horas em frente à televisão ou do computador, em invés de praticarem atividades físicas tais como brincar na rua, andar de bicicleta ou caminhar pelo bairro (Camargo et al., 2013).

$\mathrm{Na}$ adolescência e juventude, observa-se o consumo de bebidas com auto teor de sódio e açúcares, tais como os refrigerantes, acompanhados por sanduíches e hamburgeres. Isto tem se tornado um modismo nessa faixa etária que pode levar ao abandono de hábitos alimentares saudáveis (Vida \& Ferreira, 2017).

Nas pessoas adultas, Pedroni et al. (2013) destacam que a obesidade e o excesso de gordura no corpo são maiores em pessoas sedentárias, sendo que a combinação de pouca atividade física com o excesso de alimentação, ocasionam o desequilíbrio entre a energia ingerida e a gasta, favorecendo o aumento do peso.

Ao falar sobre a obesidade nas crianças, Camargo et al. (2013) explicam que além dos hábitos de vida, ela está ligada à relação mãe-filho, ou seja, a obesidade infantil se liga à obesidade materna, pois esta se torna um reflexo no hábito alimentar da família como um todo. Assim, faz-se necessário que exista a conscientização no sentido de mudança dos hábitos alimentares que proporcione a diminuição de peso, principalmente das mães. A partir daí, Vida \& Ferreira (2017) comentam que o grupo familiar é responsável por oferecer os fatores necessários à sobrevivência de um indivíduo, entre esses fatores está a necessidade básica de alimentação.

Dessa forma, além dos hábitos alimentares, no Brasil, Pedroni et al. (2013) associam a obesidade infantil e o excesso de gordura corporal ao número de refeições diárias, sendo que $51,3 \%$ das crianças que realizam de 4 ou mais refeições por dia, apresentaram menos chance de obesidade abdominal e 34,9\% apresentam menos chances de excesso de gordura.

Quanto aos riscos ocasionados pela obesidade, as pessoas obesas estão mais predispostas aos problemas cardiovasculares, especialmente os coronarianos, devido às condições como dislipidemias (níveis elevados de gorduras no sangue), hipertensão arterial, resistência à insulina e diabetes mellitus (altos índices de glicose no sangue) (Vasques et al., 2010).

Diante desses fatores, é importante compreender as relações causais da prevalência da obesidade no intuito de criar estratégias para enfrentamento do problema e desenvolver formas de monitorar casos e tendências de comportamento que levam ao problema (Pinho et al., 2013). 
De acordo com Camargo et al. (2013,) uma maneira de incentivar a mudança dos hábitos alimentares é se libertar da crença que associa magreza aos problemas de saúde e o excesso de peso à capacidade de se recuperar de doenças de maneira mais fácil. Esses valores culturais são identificados como barreiras para a adoção de comportamentos saudáveis.

Para Kovesdy, Furth e Zoccali (2016), a obesidade pode ser evitada a partir da conscientização sobre seus riscos e a adoção de um estilo de vida saudável, com nutrição adequada e a prática correta de exercícios físicos.

\section{A OBESIDADE MÓRBIDA E A CIRURGIA BARIÁTRICA}

As cirurgias voltadas ao emagrecimento surgiram na década de 1950 e tinham por objetivo reduzir a absorção dos nutrientes. Em 1956 iniciaram-se os primeiros procedimentos cirúrgicos que buscavam diminuir o tamanho do intestino delgado cuja função é absorver os alimentos. Em 1967, iniciaram-se trabalhos de restrição da capacidade gástrica observando-se mudanças nos hábitos alimentares e reduções no consumo de alimentos sólidos. No Brasil, a CB teve início no final da década de 1990, com os Doutores Garrido em São Paulo e Barroso no Rio de Janeiro (Portal Michel Menezes, 2018).

De acordo com Hintze, Bevilaqua, Pimentel e Nardo Junior (2011) "no Brasil, no ano de 1999, foram realizadas 63 gastroplastias, e, em 2003, esse número passou a ser de 2.528 intervenções: um número de CB 40 vezes maior. Nesse período, 80\% das CB foram realizadas nas regiões Sudeste e Sul".

Assim, em relação a obesidade, a Associação Brasileira para Estudo da Obesidade e da Síndrome Metabólica (ABESO, 2018) estima-se que em 2025 no mundo, cerca de 2,3 bilhões de adultos estejam com sobrepeso; e mais de 700 milhões de pessoas obesas. O número de crianças com sobrepeso e obesidade poderá atingir cerca de 75 milhões. No Brasil, aproximadamente $50 \%$ da população está acima do peso, se concentrando na faixa de sobrepeso e obesidade. Entre crianças, o número se aproxima da faixa de $15 \%$.

É importante ressaltar ainda que umas das formas de mensurar a obesidade é através do Índice de Massa Corpórea (IMC), desenvolvido por Lambert Quetelec em meados do século XIX. Para a realização deste cálculo, deve-se dividir o peso do indivíduo (em quilos) pela sua altura (em metros), elevada ao quadrado. O Portal Hospital Oswaldo Cruz (2018) explica que a partir desse índice, a obesidade é dividida em quatro níveis: sobrepeso (IMC entre 25,0 e 29,9 $\mathrm{Kg} / \mathrm{m} 2$ ), obesidade grau I (IMC entre 30,0 e $34,9 \mathrm{Kg} / \mathrm{m} 2$ ), obesidade grau II (IMC entre 35,0 e

Rev. Psicol Saúde e Debate. Dez., 2018:4(3):84-95. 
$39,9 \mathrm{Kg} / \mathrm{m} 2$ ) e, obesidade grau III (IMC maior do que 40,0 Kg/ m2). O IMC é considerado normal quando está entre 18,5 a $24,9 \mathrm{Kg} / \mathrm{m} 2$.

De acordo com a ABESO (2016), a CB não é recomendada para pacientes que se encontram no grau I de obesidade devido à incerteza dos resultados cirúrgicos, eficácia e segurança da mesma para essa população. Sendo recomendada para pacientes com IMC maior ou igual a $35 \mathrm{~kg} / \mathrm{m}^{2}$, ou seja, pacientes que se encontram no grau II (ou maior) de obesidade.

Entretanto, no grau III a cirurgia é recomendada, sendo observado um conjunto de três aspectos: prevalência elevada da compulsão alimentar, resistência aos tratamentos clínicos (com perda de peso insuficiente ou não sustentada) e associação frequente com doenças interrelacionadas, que são provocadas ou agravadas pela obesidade e que melhoram com a redução e o controle do peso (Santo \& Cecconello, 2008).

Assim, a obesidade mórbida se relaciona aos altos índices de mortalidade e à ocorrência de morbidades como diabetes, dor nos músculos e ossos, hipertensão, apneia (cessação completa e/ou redução do fluxo de ar nas vias aéreas superiores), doença cardíaca, acidente vascular cerebral e câncer (Dixon, 2010). Além destes problemas, ainda se registram os distúrbios psicossociais (sentimentos de inferioridade $\mathrm{e}$ isolamento social), geralmente ocasionados pela discriminação e preconceito (Carvalho, 2005).

Em todo o mundo percebem-se mobilizações científicas e políticas buscando combater a obesidade mórbida (Ramos, 2014). A CB é uma das formas almejadas para se tratar o problema e promover a redução de peso (Quadros, Bruscato, \& Branco, 2006), e, justifica-se pelo impacto que a doença representa, com repercussões na qualidade e na diminuição do tempo de vida das pessoas (Porto et al., 2002).

De acordo com Cruz e Morimoto (2004), para que a realização da CB seja eficaz, é necessária a avaliação por uma equipe multidisciplinar composta por cirurgião, cardiologista, pneumologista, nutricionista, psicólogo e outros profissionais, quando necessário.

A maior parte dos pacientes realiza o procedimento em busca da qualidade de vida perdida em decorrência da obesidade (Barros, Moreira, Frota, Araújo, \& Caetano, 2015). Há também aqueles que buscam melhoria no desempenho do trabalho, relacionamento social, sexualidade e rotinas diárias da vida, tais como vestir roupa e calçar sapatos (Araújo, Brito, Ferreira, Petribu, \& Mariano, 2009). De acordo com Barros et al. (2015) a cirurgia também se associa à redução da depressão e agressividade, aumento do autoconceito, sentimentos de satisfação e a capacidade de realização de atividades.

Além disso, a utilização do tratamento cirúrgico para tratar a obesidade é positiva e mostra resultado em relação à manutenção da perda de peso. Mesmo em caso de re-ganho de peso, 
registra-se que a maioria das pessoas submetidas ao tratamento não voltam aos níveis iniciais de morbidez (Santo \& Cecconello, 2008).

Porém, Zyger et al. (2016), explicam que podem ocorrer complicações após o procedimento tais como deficiência nutricionais (ferro, cálcio, vitamina $B 12$, vitamina $\mathrm{D}$, ácido fólico, zinco e albumina). Para Porto e Antoniassi Júnior (2017), outra complicação é o aumento do consumo de álcool pós-cirurgia.

Devido às complicações referentes a esta realidade, faz-se necessário, pela sua importância, buscar elucidar mais sobre as consequências no momento posterior à CB.

\section{O PÓS-OPERATÓRIO NA CIRURGIA BARIÁTRICA}

Após a realização do procedimento bariátrico, é importante que o paciente tenha consciência de que precisa se comprometer ao processo para atingir bons resultados. Neste momento, faz-se necessário obter orientações sobre como consumir a quantidade ideal de suplementos para prevenir problemas nutricionais e metabólicos (Zyger et al., 2016).

No entanto, no pós-operatório o paciente deve se conscientizar de que a obesidade se relaciona à determinados hábitos de vida, dentre eles, o comportamento alimentar que envolve o consumo de alimentos palatáveis, ou seja, são alimentos que são agradáveis ao cérebro, em que ocorre o desejo de consumi-los mesmo quando não se está com fome. É necessário se manter sempre em alerta quanto ao consumo destes alimentos, principalmente quanto à ingestão de álcool, que é processada pelo cérebro de maneira semelhante aos alimentos palatáveis (Santos \& Cruz, 2016).

Em relação ao consumo de álcool no pós-operatório da $C B$, foi realizado um estudo na Universidade Federal de Pernambuco com pacientes que se submeteram a tal procedimento. Seus resultados mostraram a prevalência do uso de álcool em períodos pré e pós-operatórios, quando comparado ao consumo da população brasileira. Porém, os autores alertam que não houve evidência de elevação do consumo em período pós-operatório (Amorim, Souza, Nascimento, Maio, \& Burgos 2015).

Outro estudo realizado na Faculdade Patos de Minas mostra casos elevados de consumo de álcool após a intercorrência. Neste estudo, infere-se que a bebida alcoólica pode ter sido utilizada para suprir a falta de alimentos bem como para promover um convívio social sem malestar e tolhimentos. Diante disso, os autores concluíram que há uma necessidade de atenção e 
cuidados intensivos aos pacientes bariátricos quanto ao abuso do álcool, pois o novo hábito pode trazer consequências nocivas à saúde (Porto \& Antoniassi Júnior, 2017).

Em seus estudos, Santos e Cruz (2016) concluíram que a CB pode alterar o metabolismo do álcool implicando em mudanças no estilo de vida e no nível de estresse. Assim, podem surgir padrões nocivos de consumo alcoólico, porém alertam para a ocorrência de fatores de risco, tais como o histórico familiar, que podem resultar em tal dependência.

Portanto, no pós-cirúrgico bariátrico há um risco de 6,5\% de se desenvolver problemas relacionados ao abuso de álcool. A justificativa para tal ocorrência é a possibilidade de transferir a compulsão pelos alimentos para o consumo abusivo alcoólico. Para os autores, houve um aumento de 7,6\% para 9,6\%, em 12 meses de pós-operatório. Esse consumo excessivo resulta em uma dificuldade no controle glicêmico. Apesar de suas conclusões, os autores alertam que há limitações nos diversos estudos encontrados na bibliografia a respeito do tema, tais como o tamanho da amostra, não sendo consideradas amostras probabilísticas ou de base populacional. Além do mais, há também o fato de grande parte dos estudos realizarem cortes transversais que inviabilizam a relação de causalidades (Silva et al., 2017).

\section{A IMPORTÂNCIA DO PSICÓLOGO NO PÓS-OPERATÓRIO DA CIRURGIA BARIÁTRICA}

O ganho de peso pode ser resultado de problemas psicológicos que influenciam o comportamento alimentar. Em alguns pacientes os problemas como o estresse, por exemplo, podem apresentar compulsão alimentar (Sousa, 2006). No mesmo sentido, os autores Porto et al. (2002) e Rosado e Monteiro (2001), explicam que o comportamento alimentar também é resultado de fatores psicológicos que podem ocasionar desequilíbrio na ingestão calórica e na diminuição do gasto de energia, favorecendo a obesidade.

O autor Halpern (1998) estabelece a relação entre o consumo de alimentos e o sistema psicobiológico. Para ele o apetite é soma dos processos que influenciam o consumo de alimentos, sendo baseado em interações do sistema psicobiológico, composto por três níveis: 1) eventos psicológicos e operações comportamentais: percepção da fome, desejo de comer, desencadeamento de sensações de prazer e bem-estar; 2) eventos fisiológicos e metabólicos; 3) eventos de interações metabólicas e de neurotransmissores do cérebro.

Diante disso, Cintra (2004) explica que para se atingir o peso adequado é necessário, além da reeducação alimentar, o acompanhamento psicológico e clínico dos pacientes. A

Rev. Psicol Saúde e Debate. Dez., 2018:4(3):84-95. 
respeito do apoio psicológico, Carvalho (2002) observa que se deve considerar todos os riscos e fatores que contribuem e predispõem o paciente à doença, de forma a buscar o equilíbrio psicológico do mesmo.

Os fatores cognitivos e comportamentais afetam direta ou indiretamente a obesidade, pois influenciam a ingestão de alimentos ou a realização de atividades físicas. Além disso, a baixa autoestima altera os níveis de ansiedade e depressão, influenciando as funções fisiológicas dos pacientes (Duchesne, 1998).

Nesse contexto, ao falar sobre o tratamento psicoterápico, Vasques, Martins e Azevedo (2004) explica que a terapia cognitiva tem apresentado mais eficácia, pois trabalha a estrutura operante do paciente procurando tratar barreiras contra a diminuição de peso e buscando comportamentos alimentares mais controlados. Além disso, a terapia cognitiva busca corrigir juntamente com o paciente, os seus pensamentos que favorecem a obesidade.

Assim, a avaliação psicológica apresenta ser fundamental tanto no pré como no pósoperatório. Aliada à avaliação e entrevistas psiquiátricas, é possível detectar psicopatologias que contribuíram para o ganho de peso (transtornos fóbico-ansiosos, depressão atípica, síndrome do comer noturno e/ou transtorno da compulsão alimentar periódica). Caso alguma psicopatologia seja detectada, é importante manter o acompanhamento psicológico, que deve atuar integrado a uma equipe multidisciplinar (Fandiño, Benchimol, Coutinho, \& Apolinário, 2004).

O acompanhamento psicológico do paciente é primordial para o sucesso da CB. Reconhecer os transtornos alimentares ligados à obesidade possibilita que o tratamento póscirúrgico seja avaliado e planejado racional e individualmente. Nesse contexto, os psicólogos podem ser instrumentos para correção de concepções equivocadas sobre as causas da obesidade, auxiliando os pacientes a estabelecerem metas razoáveis e a realizar escolhas mais saudáveis de estilo de vida, com melhoras físicas e psicológicas (Dobrow et al., 2002).

Diante do paciente que procura auxílio, o desafio do psicólogo é entender a interação dos diversos fatores psicológicos que contribuem para a obesidade e associá-los à terapia, auxiliando o paciente no manejo de seus sintomas (Vasques et. al., 2004).

\section{CONCLUSÃO}

As mudanças nos hábitos alimentares da sociedade levaram ao problema relacionado ao ganho de peso e à obesidade. Desde a infância, observa-se um desequilíbrio entre o consumo de alimentos e o gasto calórico, levando ao excesso de peso. Nos adultos, o excesso de peso é 
observado em pessoas sedentárias, que combinam a pouca atividade física com a alimentação desbalanceada.

É importante ressaltar que a obesidade leva a problemas cardiovasculares, especialmente os coronarianos. Em pacientes que se encontram em estado de obesidade a partir do grau II, com IMC entre 35,0 e 39,9 Kg/ m2, o procedimento cirúrgico já é recomendado.

Porém, é importante que o paciente tenha consciência de que os bons resultados do procedimento dependem da mudança nos hábitos de vida e alimentares e também quanto ao consumo de bebidas alcoólicas, visto que a CB pode alterar o metabolismo do álcool, implicando em mudanças no estilo de vida e no nível de estresse, podendo assim, surgir padrões nocivos de consumo alcoólico. Deve-se destacar que há estudos que apontam para uma maior busca do álcool após a CB, enquanto em outros, este dado não apareceu.

Dado o exposto, pode-se concluir que o acompanhamento psicológico do paciente aumenta as chances de sucesso da CB, considerando que o psicólogo pode ajudar a reconhecer e auxiliar nas psicopatologias que geralmente contribuem para o ganho de peso, inclusive nos transtornos alimentares em geral.

\section{REFERÊNCIAS}

Amorim, A. C. R., Souza, A. F. O., Nascimento, A. L. V., Maio, R., \& Burgos, M. G. P. A. (2015). Uso de bebida alcoólica em períodos pré e pós-operatório de cirurgia bariátrica. Rev. Col. Bras. Cir., 42(1), 03-08.

Araújo, A. A., Brito, A. M., Ferreira, M. N. L., Petribu, K. \& Mariano, M. H. A. (2009). Modificações da qualidade de vida sexual de obesos submetidos à cirurgia de FobiCapella. Ver Col Bras Cir., 36(1), 42-48.

Associação Brasileira para o Estudo da Obesidade e da Síndrome Metabólica (ABESO) (2016). Diretrizes brasileiras de obesidade. São Paulo. Recuperado em 07 de junho, 2018, de http://www.abeso.org.br/uploads/downloads/92/57fccc403e5da.pdf.

Barros, L. M., Moreira, R. A. N., Frota, N. M., Araújo, T. M. \& Caetano, J. A. (2015). Qualidade de vida entre obesos mórbidos e pacientes submetidos à cirurgia bariátrica. Rev. Eletr. Enf., 17(2), 312-321.

Camargo, A. P. P. M., Barros, A. A., Antônio, M. A. R. G. M., \& Giglio, J. S. (2013). A não percepção da obesidade pode ser um obstáculo no papel das mães de cuidar de seus filhos. Ciênc. Saúde Coletiva, 18(2), 323-333.

Carvalho, J. E. Q. (2005). Risco de doenças e custos da obesidade. In: R. S. Silva \& N. T. Ka-wara. Cuidados pré e pós-operatórios na cirurgia da obesidade (pp. 34-45). Porto Alegre: AGE.

Rev. Psicol Saúde e Debate. Dez., 2018:4(3):84-95. 
Carvalho, K. M. B. (2002). Obesidade. In: L. Cuppari, Nutrição clínica no adulto (pp. 131150). São Paulo: Manole.

Cintra, I. P. (2004). Obesidade: tratamento dietético. Revista Qualidade em Alimentação Nutrição, (17), 8-9.

Cruz, M. R. R., \& Morimoto, I. M. I. (2004). Intervenção nutricional no tratamento cirúrgico da obesidade mórbida: resultados de um protocolo diferenciado. Rev. Nutr., $\quad$ 17(2), 263-272.

Dixon, J. B. (2010). The effect of obesity on health outcomes. Molecular and Cellular Endocrinology, 316(2), 104-108. Dobrow, I. J., Kamenetz, C. \& Devlin, M. J. (2002). Aspectos psiquiátricos da obesidade. Revista Brasileira de Psiquiatria, 24(3), 63-67.

Duchesne, M. (1998). Tratamento Cognitivo-Comportamental da Obesidade. In: A. A. G. Mattos, H. L. Suplicy, M. C. Mancini \& M. T. Zanella. Obesidade (pp. 261- 274). São Paulo: Lemos Editorial.

Fandiño, J., Benchimol, A. K. Coutinho, W. F., \& Apolinário, J. C. (2004). Cirurgia bariátrica: aspectos clínicos-cirúrgicos e psiquiátricos. Revista de Psiquiatria do Rio Grande do Sul, 26(1), 47-51.

Halpern, A. (1998). Obesidade, mitos e verdades. São Paulo: Contexto.

Hintze, L., J., Bevilaqua, C. A., Pimentel, E. B., \& Nardo, N. Jr. (2011). Cirurgia Bariátrica no Brasil. Rev. Ciênc. Méd., Campinas, 20(3), 87-98.

Kovesdy, C. P., Furth, S. L. \& Zoccali, C. (2016). Obesidade e doença renal: $\quad$ consequências ocultas da epidemia. J Bras Nefrol, 39(1), 01-10.

Organização das Nações Unidas para a Alimentação e a Agricultura e Organização PanAmericana da Saúde (2016). Panorama da Segurança Alimentar e Nutricional na América Latina e Caribe. Recuperado em 07 de junho, 2018, de http://www.fao.org/3/ai69770.pdf.

Pedroni, J. L., Rech, R. R., Halpern, R., Marin, S., Roth, L. R., \& Sirtoli, M. et al. (2013). Prevalência de obesidade abdominal e excesso de gordura em escolares de uma cidade serrana no sul do Brasil. Ciênc. Saúde Coletiva, 18(5), 1417-1425.

Pinho, C. P. S., Diniz, A. S., Arruda, I. K. G., Batista Filho, M., Coelho, P. C., \& Sequeira, L. A. S. et al. (2013). Prevalência e fatores associados à obesidade abdominal em indivíduos na faixa etária de 25 a 59 anos do Estado de Pernambuco, Brasil. Cad. Saúde Pública, 29(2), 313-324.

Portal Hospital Oswaldo cruz. Recuperado em 07 de junho, 2018 de http://centrodeobesidadeediabetes.org.br/tudo-sobre-obesidade/tratamento-daobesidade/.

Portal Michel Menezes. Recuperado em 07 de junho, 2018 de https://aobesidade.com.br/historia-da-cirurgia-bariatrica/

Rev. Psicol Saúde e Debate. Dez., 2018:4(3):84-95. 
Porto, D. L., \& Antoniassi, G. Jr. (2017). A decorrência do uso do álcool em pacientes que submeteram à Cirurgia bariátrica. Rev Psicol Saúde em Debate, 3(1), 14-16.

Porto, M. C. V., Brito I. C. B., Calfa, A. D. F., Amoras, M., Villela, N. B., \& Araújo, L. M. B. (2002). Perfil de obeso classe III do ambulatório de obesidade de um hospital universitário de Salvador, Bahia. Arq Bras Endocrinol Metab, 46(6), 668-673.

Quadros, M. R. R., Bruscato, G. T. \& Branco, A. J. F. (2006). Compulsão alimentar em pacientes no pré-operatório de cirurgia bariátrica. Psicol. Argum, 24(45), 59-65.

Ramos, A. C. (2014). O Brasil procurando preencher o seu espaço na cirurgia bariátrica. $A B C D$ Arq Bras Cir Dig., 27(1), 1.

Rosado, E. L., \& Monteiro, J. B. R. (2001). Obesity and diet macronutrients substitution. Rev. . 14(2), 145-152.

Santos, J. G., \& Cruz, M. S. (2016). Alcoolismo após cirurgia bariátrica: relato de caso. J. bras. Psiquiat., 65(4), 340-343.

Santo, M. A., \& Cecconello, I. (2008). Obesidade mórbida: controle dos riscos. Arq. Gastroenterol., 45(1), 01-02.

Silva, S., Milheiro, A., Ferreira, L., Rosete, M., Campos, J. C., \& Almeida, J., et al. $\quad$ (2017). Gastrectomia Vertical Calibrada no tratamento da obesidade mórbida. Resultados a longo prazo, comorbidades e qualidade de vida. Revista Portuguesa de Cirurgia, 40,1120.

Sousa, V. J. (2006). Hábito alimentar de pacientes obesos mórbidos. Dissertação de Mestrado em Qualidade em Alimentos, Universidade de Brasília, Brasília, DF.

Vasques, A. C. J., Priore, S. E., Rosado, L. E. F. P. L., \& Franceschini, S. C. C. (2010). Utilização de medidas antropométricas para a avaliação do acúmulo de gordura visceral. Rev. Nutr., Campinas, 23(1), 107-118.

Vasques, F., Martins, F. C., \& Azevedo, A. P. (2004). Aspectos psiquiátricos no tratamento da obesidade. Revista Brasileira de Psiquiatria Clínica, 31(4), 195-198.

Velásquez-Meléndez, G., Pimenta, A. M. \& Kac, G. (2004). Epidemiologia do sobrepeso e da obesidade e seus fatores determinantes em Belo Horizonte (MG), Brasil: estudo transversal de base populacional. Rev Panam Salud Publica, 16(5), 308-314.

Zyger, L. T., Zanardo, V. P. S., \& Tomicki, C. (2016). Perfil nutricional e estilo de vida de pacientes pré e pós-cirurgia bariátrica. Sci Med., 26(3), 1-8.

Vida, P., \& Ferreira, L. (2017). Obesidade mórbida: a família como cúmplice. Rev Psicol Saúde em Debate, 3 (Supl. 1), 44-45. 